REVISTA

MEXICANA DE

ECONOMÍA Y

FINANZAS

REMEF

(THE MEXICAN JOURNAL OF

ECONOMICS AND FINANCE)
Revista Mexicana de Economía y Finanzas, Nueva Época

Volumen 17 Número 1, Enero - Marzo 2022, pp. 1-18, e716

DOI: https://doi.org/10.21919/remef.v17i1.716

(Recibido: 17/junio/2020, aceptado: 14/diciembre/2020, publicado: 19/octubre/2021)

\title{
Inclusión financiera en jóvenes universitarios en México, 2017-2018
}

\author{
Gustavo León Cuanalo $^{1}$ - Benemérita Universidad Autónoma de Puebla, México \\ Ariadna Hernández Rivera ${ }^{2}$ - Benemérita Universidad Autónoma de Puebla, México \\ Gonzalo Haro Álvarez ${ }^{3}$ - Benemérita Universidad Autónoma de Puebla, México
}

En México existen programas para el acceso a Servicios y Productos Financieros en diferentes grupos poblacionales, sin embargo, los Jóvenes Universitarios de las áreas económico-administrativas tienen una Inclusión Financiera precaria, a pesar de que ellos cuentan con capacitación e instrucción en su formación académica para participar en el mercado financiero. La presente investigación pretende ser un nuevo aporte en el tema, ya que se analizó a los estudiantes de doce universidades, de las cinco zonas metropolitanas con mayor concentración poblacional de México, con el objetivo de estimar los niveles y factores que limitan o promueven la inclusión financiera. Utilizando el Modelo Scoring, basado en técnicas estadísticas y econométricas; obteniendo las desigualdades que existen entre Estados y Zonas. Se sugieren a las instituciones de educación superior, adaptar los planes de estudio a esquemas prácticos para el día con día; ya que una mayor inclusión implica una puerta de acceso a diferentes transacciones económicas. Entre las conclusiones a destacar: se encuentra que, los lugares con mayor desarrollo económico son más factibles a estar incluidos financieramente.

Clasificación JEL: A22, D14, G2, G53, I23.

Palabras clave: Inclusión Financiera, Educación Financiera, Jóvenes Universitarios, Mercado Financiero; Servicios y Productos Financieros.

\section{Financial Inclusion in Bachelor Students in Mexico, 2017-2018}

In Mexico there are programs for access to Financial Services and Products in different population groups, however. Bachelor students from economic-administrative areas have a precarious Financial Inclusion, even though they have training and instruction in their academic training to participate in the financial market. This research aims to be a new contribution on the subject, since students from twelve universities, from the five metropolitan areas with the highest population concentration in Mexico, were analyzed, with the aim of estimating the levels and factors that limit or promote inclusion financial Through the Scoring Model, based on statistical and econometric techniques; obtaining the inequalities that exist between States and Zones. Higher education institutions are suggested to adapt the study plans to practical schemes for the day to day, since greater inclusion implies a gateway to different economic transactions. Among the conclusions to be highlighted: it is found that the places with the greatest economic development are more likely to be included financially.

JEL Classification: C61, C63, G12, G17, G14.

Keywords: Bachelor students, Financial inclusion, Financial market, Financial education, Financial products and services.

${ }^{1}$ Autor de correspondencia. Boulevard 22 Sur, Cd Universitaria, Col. San Manuel. C.P. 72592 Puebla, Pue. Tel. 52222229

5500 ext. 7800. Correo electrónico: lecg994@gmail.com; https://orcid.org/0000-0003-2619-6374

${ }^{2}$ Correo electrónico: ary_hr@yahoo.com.mx; ORCID: https://orcid.org/0000-0003-2377-145X

${ }^{3}$ Correo electrónico: gonzalo.haro@correo.buap.mx; ORCID: https://orcid.org/0000-0002-8440-5966

*Sin fuente de financiamiento para el desarrollo de la investigación 


\section{Introducción}

La falta de inclusión financiera es resultado de diversos factores y circunstancias, como; la situación económica, el nivel educativo, género, área geográfica, edad, grado de desarrollo, desigualdad social entre otros. En México, la exclusión es un problema frecuente en gran parte del país, se da en cualquier condición social, inclusive dentro de las zonas urbanas y metropolitanas, estando presente en los diferentes segmentos de la población, como jóvenes universitarios, adultos mayores, personas con un ingreso salarial bajo, etc.

Datos del Banco Mundial (2018), muestran que alrededor de 2.5 mil millones de individuos en el mundo, viven destituidos del sistema financiero. Este número se encuentra en las localidades más vulnerables, que residen en comunidades rurales y países en desarrollo. Es necesario reconocer que, pese a los esfuerzos de organismos internacionales, gobiernos y bancos centrales interesados en el tema del acceso y uso de los servicios financieros, la generación de datos e indicadores basados en encuestas sobre inclusión financiera se encuentra limitada a un grupo reducido de países (Banco Interamericano de Desarrollo, 2015).

Por otro lado, existe una parte de la población que ha sido instruida en las Universidades en las áreas económico-administrativas, sin embargo, a pesar de ello, los estudiantes no han conseguido aterrizar los conceptos teóricos a la práctica, como lo demuestran los estudios de Hernández-Rivera (2019; y 2020); Hernández-Rivera y Rendón (2021a); así como Osorno y Hernández-Rivera (2021b). Estas investigaciones exponen que los jóvenes aún no logran incluirse financieramente en el sistema, a pesar de que han tenido capacitación formal en estas temáticas. En este trabajo, se propone un Modelo Scoring para estimar la categorización de la inclusión financiera en los diferentes estados y zonas metropolitanas del país; con el objetivo de explicar la construcción de un modelo mediante análisis estadístico y econométrico similar al elaborado por Ochoa, Galeano, Agudelo (2010); y Rayo, Lara, Camino (2010).

Por ello, es necesario estudiar la inclusión financiera, debido a que es un aspecto fundamental para construir un país que necesita mejores condiciones para forjar un mayor desarrollo, equitativo y acciones muy concretas para el bienestar de la población en general, considerando como un sector importante a las nuevas generaciones en los ámbitos de competencia e innovación tecnológica (Hernández-Rivera, 2020).

Existen varios estudios que reflejan la falta de inclusión financiera, capacidades, conocimientos, comportamientos y otras variables (Peña, Hoyo y Tuesta, 2014; Cámara, Peña y Tuesta, 2013; Índice de Inclusión Financiera Citibanamex, 2019), adicionalmente se encuentran diversos reportes que muestran la situación actual de México como la Encuesta Nacional de Inclusión Financiera (2018); Boletín de Inclusión Financiera (CNBV, 2019); El Ahorro en México: Productos, Instrumentos y Evolución (CNBV, 2021), entre otros.

Sin embargo, en México no se encontró un estudio sobre Inclusión Financiera que reuniera a una muestra de estudiantes universitarios de áreas económico-administrativas de zonas metropolitanas, por lo que esta investigación pretende ser pionera en este tipo de análisis que se enfocan en grupos específicos de jóvenes que tienen conocimientos teóricos, pero no necesariamente prácticos y que muchos de ellos, aún no comprenden el funcionamiento, costos-beneficios, riesgos, etcétera de los servicios y productos financieros disponibles en el mercado. 
Según datos de la Encuesta Nacional de la Dinámica Demográfica (ENADID, 2018), los jóvenes representan el $24.6 \%$ de la población joven; es decir hay 30.7 millones de personas que oscilan entre los 15 y 29 de edad. Para el presente estudio, se pretende alcanzar una aproximación sobre el nivel de inclusión financiera de los jóvenes universitarios de las cinco zonas metropolitanas más pobladas en México (SEGOB, SEDATU, CONAPO e INEGI, 2018): 1. Valle de México; 2. Guadalajara; 3. Monterrey; 4. Puebla - Tlaxcala; y 5. Toluca, que representan el 45\% de la población estudiantil (1’894,616), según datos de la Asociación Nacional de Universidades e Instituciones de Educación Superior (ANUIES, 2020).

\section{Revisión de la literatura.}

La inclusión financiera se define como el acceso y uso de servicios financieros formales que están disponibles en el mercado, bajo una adecuada supervisión, con la finalidad de mejorar las capacidades de todas las personas y empresas, tomando en cuenta cuatro pilares fundamentales: acceso, uso, protección al consumidor y educación financiera (Consejo Nacional de Inclusión Financiera, CONAIF, 2016).

La inclusión financiera logra que los países puedan tener expectativas de una competencia en el mercado financiero, accediendo a los establecimientos mercantiles y no mercantiles, ofrecer y prestar servicios, logrando tanto la cobertura como el acceso a más usuarios (Banco Mundial, 2018; y CONEVAL, 2019). Para ampliar el alcance del sistema financiero, se requiere de la iniciativa pública y privada, que incluya la relación entre los ofertantes (instituciones financieras) y los demandantes (sociedad, hogares, pequeñas y medianas empresas); dependiendo de sus necesidades (CEPAL, 2018).

La relación entre inclusión y educación puede equilibrar el sistema financiero de un país, al ampliar la base de usuarios (CEMLA, 2014). Sin embargo, la educación financiera demanda la intervención de los agentes implicados: gobierno, sociedad e instituciones en conjunto, para ejercer su uso y funcionamiento (Home, 2016).

La inclusión financiera de un país se puede considerar un elemento esencial que propicia el desarrollo económico, tanto a nivel nacional como de manera individual e incluso puede contribuir a una mayor solvencia de los mercados financieros (CEPAL, 2018). Lo anterior permite a las personas tomar mejores decisiones con respecto a sus finanzas y mejorar su nivel de vida (Rivera y Bernal, 2018).

El acceso a servicios y productos financieros permite que tanto sujetos, como sociedades: vigilen, gestionen o aumenten su dominio, para poder efectuar eficientes actividades comerciales, tomar disposiciones de ahorro y capitalizar sus regímenes de consumo e inversión (Sánchez, 2013; Ley de Ahorro y Crédito Popular, 10/01/2014). Esto fomenta el incremento para el uso y el acceso a estos servicios y productos con la finalidad de que impacten positivamente en los ingresos de los hogares (Guízar, González-Vega y Miranda, 2015).

Li, Espinosa, Cárdenas \& Serrano (2018), coinciden que existe un bajo uso de servicios y productos financieros formales en México; únicamente el 31\% de la población total, posee una tarjeta de crédito, $25 \%$ un seguro y $40 \%$ una cuenta de ahorro para el retiro. Lo que significa que el resto de los agentes económicos, se hallan excluidos, al no contar con ninguno de estos servicios mencionados. 
En los últimos años se ha suscitado un mayor interés en la inclusión financiera (Schröeder, 2017), una persona que no se inmerge al mercado financiero, es difícil que entienda, lo que este tiene para ofrecerle. En todo momento se toman decisiones relacionadas con la seguridad económica, como: pensiones, hipotecas, tarjetas de crédito, la compra de un auto, de una casa, entre otros, por ello, se debe estar dentro del sistema financiero, así como invertir en educación para elegir de manera consciente (Hernández-Rivera, 2019 y 2020).

Varios autores como Sarma y País (2010), Lusardi (2011), Racanéelo (2014) Villagómez (2016), y Hernández-Rivera (2019), concuerdan que la inclusión a los mercados financieros se torna más compleja para los individuos que no están lo suficientemente preparados para tomar decisiones acertadas. Debido a la falta de conocimientos sobre conceptos en materia de educación financiera por parte de la población provoca menor uso de Productos y Servicios Financiero (Rivera y Bernal, 2018).

Esta inclusión al Mercado Financiero ayuda a mejorar los ingresos de los usuarios, así como también propicia: mayores oportunidades a través de la educación financiera; aumento del gasto; esquemas de consumo, bienestar y entretenimiento (Klapper \& Hess, 2016). Lo que permite desarrollar habilidades para la vida diaria, como son: el presupuestar los gastos, elegir adecuadamente una tarjeta de crédito, contratar algún seguro (automotriz, gastos médicos, hogar), contar con un fondo de emergencia y ahorrar para el retiro (Hernández, 2014).

Pero esta inclusión a los mercados financieros, a pesar de estar más presentes en las zonas urbanas y metropolitanas de México, no logra cubrir a toda la población que habita estas regiones, dejando fuera principalmente a los jóvenes, quienes representan un 31\% de la población total del país: es decir, casi la tercera parte del total de habitantes de México (IMJUVE, 2020). Esta exclusión trae como consecuencia bajos niveles de bancarización en la población estudiantil, pero a su vez se plantea el desafío de incrementar la oferta de servicios y productos financieros hacia este nuevo mercado que son los jóvenes universitarios, fomentando procesos de digitalización para facilitar sus operaciones financieras (Reyna, 2020).

Como lo explican Vargas y Reyes (2019), la tecnología es una herramienta que permite a los jóvenes interactuar fácilmente con el mercado financiero. La banca en línea es la más común, porque ellos tienen acceso a un dispositivo móvil, y les es posible realizar sus transacciones bancarias más rápido, fácil y eficazmente (Escalera, Tejada y García, 2017; y Osorno y Hernández-Rivera, 2021), teniendo como factor importante la funcionalidad y transparencia del sistema financiero, para que las sociedades bancarias oferten mejores opciones de financiamiento a los individuos (Lecuona, 2017).

El uso de las Tecnologías de la Información y la Comunicación (TIC's), generan que cada vez más jóvenes se encuentren inmersos en el sistema financiero (Calderón, Carvajal, Leiva; 2014). Estas tecnologías contribuyen a mejorar el acceso al mercado financiero, por ello, en México se ha tratado de regular a los establecimientos de tecnología aplicada a las Finanzas, a través de Ley Fintech (DOF, 09/03/2018), que tiene el objetivo de acompañar el proceso de innovación tecnológica, así como el desarrollo del mercado financiero.

Derivado de lo anterior, el crecimiento del sector financiero a consecuencia de la participación de los jóvenes universitarios denota un aumento en la innovación de los servicios y productos financieros (por ejemplo, el uso de las Fintech), propiciando que se expanda su uso para 
poder llegar a más sectores o grupos poblacionales (Díaz, 2020). Los jóvenes son los más beneficiados, ya que ellos son los principales consumidores de estas tecnologías. Peterson (2018) argumenta que las finanzas digitales tienen algunos beneficios: pueden conducir a una mayor inclusión financiera, la expansión de los servicios financieros a los sectores no financieros y la extensión de los servicios básicos a las personas, ya que casi el 50\% de los jóvenes a nivel mundial poseen un teléfono móvil.

Autores como Lusardi y Mitchel (2011), plantean que, el conocimiento financiero es fundamental para los individuos en la edad productiva (adolescentes y jóvenes), porque permite tener una mayor conciencia, que ayuda a una mejor administración de los recursos, participando en inversiones y el ahorro para el futuro. Algunos de los contrastes más significativos en la enseñanza para los jóvenes a partir de los 15 años, indican que no a todos se les ofrece la misma oportunidad de desarrollar su educación financiera (Hernández-Rivera, 2020), con el único fin de lograr que los jóvenes universitarios comprendan el impacto de sus decisiones financieras (Zapata, Cabrera, Hernández, y Martínez, 2016).

Por su parte, la CNBV en México, en colaboración en el INEGI, efectuaron la tercera Encuesta Nacional de Inclusión Financiera (ENIF, 2018) aplicada a toda la población en general. Con la finalidad de observar las adversidades que afronta la población para integrarse al sistema financiero, crear información estadística y guías para diseñar políticas gubernamentales e instaurar términos en materia de inclusión financiera.

Así mismo el Índice de Inclusión Financiera de Citibanamex (2019), muestra los alcances y avances de la población total a nivel estatal y municipal en México, haciendo énfasis en los indicadores de inclusión, permitiendo contrastar progresos de un año a otro. También identifica cuáles son las entidades federativas y municipios con mayor inclusión, diferencia las zonas donde los avances son más notables sobre aquellas donde existe el rezago y la exclusión.

El porcentaje de adultos con una cuenta bancaria o un crédito en una institución bancaria formal oscila entre el 44\% y 58\% (CONAIF, 2016), sin embargo, estos productos financieros no están enfocados exclusivamente en los jóvenes, por tanto, deben ser visibilizados como sector relevante. Escalera, Tejada, y García (2017) argumentan que deben ser considerados financieramente para desarrollar el conocimiento y tomar decisiones inteligentes a través de la educación financiera.

Es por ello por lo que un sistema financiero inclusivo se ha convertido en una prioridad política en muchos países. Iniciativas para disminuir la exclusión han venido de los reguladores financieros, gobiernos y las industrias bancarias. En algunos países se iniciaron medidas legislativas como en Francia con la ley de exclusión, que enfatizó el derecho de una persona a tener una cuenta bancaria de ahorro (Sarma y Pais, 2010). Rincón (2019) infiere que las cuentas de ahorro se derivan de la combinación entre baja inflación y alto crecimiento económico, en un contexto macroeconómico (Guimaraes, 2020).

Las cuentas de ahorro son el servicio financiero más utilizado por los jóvenes universitarios, lo que les permite estar incluidos de forma directa o indirecta con alguna institución bancaria formal, esto trae consigo beneficios desde una perspectiva macroeconómica como las altas tasas de participación en el mercado financiero, así lo explican autores como Arango y Cardona (2019), y Bermeo et al (2019).

Morales (2019), plantea que los jóvenes que empiezan a laborar durante su vida universitaria deben estar más capacitados, no solo académicamente sino también financieramente. Los 
estudiantes durante su preparación universitaria empiezan a ser independientes, lo que implica que deben estar protegidos, por ello una pieza fundamental para la protección son los "seguros" que ofrecen las instituciones financieras.

Por ejemplo, los seguros de gastos médicos amparan a los jóvenes por cualquier causa o imprevisto, transferir una parte o la totalidad del riesgo a una entidad a cambio de una prima, es una estrategia de protección y respaldo que le puede ayudar a tener una mayor estabilidad financiera (Argüello, 2018). Asimismo, un seguro de hogar se hace cuando un universitario es consciente de los riesgos a los que se está expuesto diariamente, sin embargo, poco se tiene en cuenta que el tiempo es un factor que también juega un papel muy importante (Fajardo, Camilla, Uribe y Paipa, 2018)

También hay que considerar los seguros educativos, especialmente durante la vida universitaria ya que son una alternativa para hacer frente a los gastos y eventualidades. Se trata de un producto financiero en el que se paga una prima o cantidad mensual, trimestral, semestral o anual (CONDUSEF, 2019 b).

Adicionalmente el Banco de España (2011) aconseja a toda la población, acumular un fondo de emergencia equivalente a tres y seis meses de gastos normales, es decir, lo suficiente para pagar la hipoteca o el alquiler y la cuota de los demás préstamos, comida, luz, seguros, colegios, etc. durante ese periodo.

Considerando que los universitarios también adquieren medios de transporte como el automóvil, el seguro de auto es usado para dar el soporte y la atención debida en el menor tiempo posible a los jóvenes ante cualquier siniestro o percance vehicular, al ocurrir un accidente de cualquier gravedad (Vera, 2019).

Además, se debe tener una AFORE (Ahorro de Fondo para el Retiro) que es un instrumento que le permite a los jóvenes cumplir con uno de sus objetivos primordiales, contar con recursos suficientes en el momento de su retiro laboral, esperando que en su vejez sea una etapa plena en donde se dediquen a efectuar proyectos personales como viajar, realizar alguna actividad de esparcimiento o simplemente descansar (Ordaz, Pacheco, Guillén y Gutiérrez, 2019).

Estos estudios y acciones señalados anteriormente se han visto reflejadas de forma positiva en un aumento de la inclusión financiera. Las instituciones financieras que ofrecen servicios también han aumentado, al igual que los puntos de acceso.

\section{Inclusión Financiera en los Jóvenes Universitarios en México.}

México se ha convertido en un caso interesante para estudiar la inclusión financiera, ya que cuenta con trayectoria en su evolución regulatoria, así como las instituciones financieras y los servicios ofertados a los distintos sectores de la población con bajos ingresos (Villacorta y Reyes, 2012). El Gobierno Mexicano, ha estado promoviendo la educación financiera a través de programas sociales, con la finalidad de desarrollar un sistema financiero equilibrado y equitativo (CONAIF, 2017).

El Informe de Competitividad Global 2018 del Foro Económico Mundial, señaló que el sistema financiero mexicano ha mejorado, logrando posicionarse en el lugar 61, de 140 países analizados (RNIF, 2018) destacando que, en 2017, el $97.8 \%$ de la población adulta reside en una zona geográfica con al menos un punto de acceso para depósitos y retiros. 
La Comisión Nacional Bancaria y de Valores (CNVB, 2016), explicó que el acceso y uso de los servicios y productos financieros, están regulados por Órganos Gubernamentales a través del Banco de México (BANXICO, 2018), cuya finalidad se enfoca en garantizar la protección y defensa del consumidor, promoviendo las capacidades económicas de los diferentes tipos de segmentos de la población para poder lograr cobertura en inclusión y desarrollo del sistema financiero del país.

Los servicios y productos financieros cada vez son más numerosos y complejos; lamentablemente ciertos sectores los utilizan de manera incorrecta e inadecuada, siendo algunas personas rezagadas por la falta conocimiento. De acuerdo con la Oficina de Información Científica y Tecnológica para el Congreso de la Unión (INCyTU, 2018), en México, el 30\% de sus habitantes posee nociones básicas de educación financiera, en contraste con otras naciones como: Noruega, Dinamarca y Suecia (los tres países con 71\%); Reino Unido (67\%) y Canadá (68\%).

Por su parte, el bajo nivel de ingresos de los jóvenes en México obliga a los universitarios a realizar un doble rol en la sociedad (estudiar y trabajar), integrado el ámbito académico con el laboral, con el propósito de conseguir los recursos necesarios para sustentarse (Osorno y HernándezRivera, 2021). Sin embargo, a pesar de que muchos de ellos, tienen un ingreso, aún no han logrado incorporarse al Sistema Financiero.

Son diversos los factores que excluyen a la población joven en México del acceso a servicios y productos financieros, debido a que regularmente durante esta etapa no cuentan con ingresos fijos y casi siempre hay dependencia económica de los padres. De acuerdo con la Procuraduría Federal del Consumidor (PROFECO, 2017), existen jóvenes que ya toman sus propias decisiones financieras; aunque algunos con problemáticas o limitantes diversas como: a) la insuficiente búsqueda de información, b) Las restricciones de los bancos comerciales que limitan sus servicios y productos financieros por falta de ingresos, y c) Reformas o Leyes que regulan el mercado financiero, que aún no son incluyentes.

Aunado a esto, Salazar, Rodríguez y Jaramillo (2017); resaltan la importancia e interés de la inclusión financiera en un contexto social, como medio para acceder a una mejor calidad de vida. Por ello se han realizado diversas acciones para promover la inclusión y educación financiera a través de programas que coadyuven a la integración de los jóvenes al sistema financiero mexicano. Por ejemplo, el Programa Nacional de Juventud 2014-2018 (DOF, 30/04/2014), del Instituto Mexicano de la Juventud (IMJUVE), que ha contribuido al fomento para la educación de los jóvenes, con la finalidad de que ellos tomen decisiones responsables para no endeudarse, así como crear y fortalecer un patrimonio propio.

En México como lo explica Ceballos y Santiago (2019), existe una acelerada expansión de algunos servicios financieros mediante procesos como la bancarización inducida o la misma dinámica de mercado de servicios como las tarjetas de crédito. La inclusión financiera podría ser una estrategia de desarrollo con notoriedad a nivel nacional desde la perspectiva de política pública, económica y financiera.

Por otra parte, en la administración del presidente Andrés Manuel López Obrador (AMLO, 2018 - 2024), se estima que el Programa “Jóvenes Construyendo el Futuro" permitirá la inclusión de más de 900 mil individuos, en un rango de 18 - 29 años, que podrán ser bancarizados para recibir su beca mensual de manera directa, a través la Secretaría del Trabajo y Previsión Social (STPS, 2019). Esta es una acción, podría promover el desarrollo de las finanzas personales de los jóvenes. 
Asimismo, las Instituciones Bancarias Privadas en México como: Banco Santander, CITI Banamex y Banco Azteca, han abierto su mercado a sectores de la población que antes no tenían contemplados, por ejemplo, los jóvenes universitarios. La oferta de servicios y productos financieros ha crecido en este segmento que concentra a los universitarios, quienes inician su vida laboral e historial crediticio (CONDUSEF, 2018). Con el principal objetivo de incluirlos a nuevas modalidades de crédito, para adquirir diferentes bienes o servicios: auto, casa, etc.

El Gobierno de México a través de Banxico (2019) implementó CoDI (Forma de Cobro Digital), que tiene un conjunto de códigos QR y NFC (Código de barras bidimensional cuadrado), para facilitar que tanto negocios como beneficiarios, logren ejecutar oportunidades de compra / venta sin dinero en efectivo. Las instituciones participantes del Sistema de Pagos Electrónicos Interbancarios (SPEI), los comercios electrónicos, proveedores de servicios, serán parte de esta nueva forma de pago. Entre los beneficiados de esta nueva modalidad se encuentran los adolescentes y jóvenes, quienes están inmersos con los avances y usos de las Nuevas Tecnologías (CONDUSEF, 2019).

Por su parte, el Banco de México (2020) presentó una iniciativa para que los menores de edad puedan abrir cuentas bancarias para incluirse al sistema financiero, así como fomentar el ahorro sin necesidad de un tercero o representante, con la finalidad de recibir recursos derivados de programas gubernamentales y transferencias electrónicas. Lo que permitirá evitar el mal uso de estas cuentas de ahorro, así como verificar la legalidad y autenticidad de los fondos respectivos.

Por estas razones, la investigación busca medir y analizar los niveles de inclusión financiera en los jóvenes universitarios que cursan alguna licenciatura de áreas económico-administrativas en las principales zonas metropolitanas de México, quienes se suponen deberían estar más preparados académicamente en estos temas, lo cual se pretende demostrar si esta condición se cumple o no.

\section{Metodología}

\subsection{Selección de la muestra}

El estudio corresponde a una muestra de 3,600 estudiantes, de áreas Económica-administrativas de Instituciones de Educación Superior Públicas y Privadas, de los cuales 51\% son hombres y 49\% son mujeres, que oscilan en el rango de edad, entre los 17 - 25 años. El instrumento se aplicó entre los años 2017-2018 en los estados que pertenecen a las cinco primeras Zonas Metropolitanas (ZM) de México:1. Valle de México (VM); 2. Guadalajara; 3. Monterrey; 4. Puebla - Tlaxcala; y 5. Toluca. La Tabla 1, muestra los estudiantes por estado que fueron considerados en el estudio. 
Tabla 1. Estudiantes encuestados por Entidad Federativa.

\begin{tabular}{|l|c|c|c|}
\hline Zona Metropolitana & Entidad Federativa & Estudiantes & Porcentaje \\
\hline 1. Valle de México & Ciudad de México & 471,696 & $25 \%$ \\
\hline 2. Guadalajara & Jalisco & 255,630 & $13 \%$ \\
\hline 3. Monterrey & Nuevo León & 211,676 & $11 \%$ \\
\hline 4. Puebla - Tlaxcala & Puebla & 264,196 & $14 \%$ \\
\cline { 3 - 4 } & Tlaxcala & 34,484 & $2 \%$ \\
\hline 5. Toluca & Estado de México & 659,069 & $35 \%$ \\
\hline
\end{tabular}

Fuente: Elaboración propia con base en trabajo de campo.

Para que la muestra fuese representativa, se consideró un nivel de confianza del 95\% con un error muestral deseado del 1.6319\%, misma que arrojó una $n=3,600$. El muestreo utilizado fue en primera instancia por estratificado y seguido conglomerado.

Se seleccionaron a las mejores universidades con la finalidad de encontrar estudiantes que tuviesen una mayor instrucción académica en Educación e Inclusión Financiera. En primera instancia se seleccionaron las primeras 50 posiciones de las Universidades que se encontraban entre las mejores rankeadas (ver tabla 2), de acuerdo con la revista América Economía (2018). Posteriormente se enviaron solicitudes de aplicación de la encuesta a las instituciones, y se llevó a cabo el proceso únicamente en las Facultades, Institutos o Departamentos que permitieron ser evaluados.

Tabla 2. Mejores Universidades de México 2018.

\begin{tabular}{|l|c|}
\multicolumn{1}{|c|}{ Institución } & $\begin{array}{c}\text { Revista América Economía } \\
\mathbf{2 0 1 8}\end{array}$ \\
\hline $\begin{array}{l}\text { Universidad Nacional Autónoma de México UNAM } \\
\text { Facultad de Estudios Superiores Aragón } \\
\text { Facultad de Estudios Superiores Acatlán }\end{array}$ & 1 \\
\hline $\begin{array}{l}\text { Instituto Tecnológico y de Estudios Superiores } \\
\text { de Monterrey ITESM Campus Monterrey }\end{array}$ & 2 \\
\hline Instituto Politécnico Nacional IPN & 3 \\
\hline Universidad Autónoma Metropolitana UAM & 4 \\
\hline Universidad de Guadalajara UDG & 5 \\
\hline Universidad Autónoma de Nuevo León UANL & 6 \\
\hline Benemérita Universidad Autónoma de Puebla BUAP & 9 \\
\hline Universidad Iberoamericana IBERO & 11 \\
\hline Universidad Autónoma del Estado de México UAEMEX & 18 \\
\hline Universidad de Monterrey UDEM & 21 \\
\hline $\begin{array}{l}\text { Universidad Popular Autónoma del Estado de } \\
\text { Puebla UPAEP }\end{array}$ & 23 \\
\hline Universidad Autónoma de Tlaxcala UATX & 46 \\
\hline
\end{tabular}

Fuente: Elaboración propia con datos de América Economía (2018).

El método de recolección fue la encuesta, teniendo por instrumento el cuestionario, que contempló 36 indicadores en escala dicotómica, ordinal, nominal y likert (los cuales por la naturaleza de la pregunta se categorizaron de forma descendente, llevando a obtener una cifra, la cual se utiliza 
a favor de la inclusión financiera.) sustentadas en literatura relacionada con la temática y la observación de evidencias.

Para la construcción del instrumento se consideraron las variables más representativas de acuerdo con diversos autores (Lusardi; 2011, Racanéelo y Herrera; 2014, Villagómez; 2016, BANXICO; 2018) con la finalidad de obtener el nivel de inclusión financiera de los jóvenes universitarios.

Esta técnica es ampliamente utilizada como procedimiento de investigación, ya que permite obtener y elaborar datos de modo rápido y eficaz a comparación de otros. En el ámbito económico, financiero y educativo, son muy numerosas las investigaciones realizadas utilizando esta técnica, como queda demostrado en los censos poblaciones y económicos realizados por el INEGI (2018), encuestas de inclusión financiera por CNBV (2018).

\subsection{El modelo propuesto}

El Modelo Scoring, trata de obtener a partir la relación existente entre diversas variables que definen tanto al solicitante como a la operación, una regla general que permita determinar, con rapidez y fiabilidad, la probabilidad de fallido de una determinada solicitud (Ratner, 2012). Con este análisis se obtiene el puntaje de inclusión financiera (por individuo) entre zonas metropolitanas y estados a partir de la siguiente expresión:

$$
\text { Scoring Inclusion Financiera }(I F)=\alpha+B_{1} H C F+B_{2} U S P Y S F+B_{3} H C
$$

\section{Donde:}

Scoring Inclusión Financiera (IF)= La variable dependiente que se requiere explicar.

Hábitos y comportamientos financieros $(H C F)=$ La variable que engloba los hábitos $\mathrm{y}$ comportamientos financieros de los estudiantes.

Uso de productos y servicios financieros (USPYSF) = La variable que engloba el uso de productos y servicios financieros de los estudiantes.

Habilidades y conocimientos que tienen los jóvenes universitarios $(\mathrm{HC})$ = La variable que engloba las habilidades y conocimientos que tienen los jóvenes universitarios.

En la tabla 3, se exponen las variables utilizadas para la construcción del modelo, considerando los diferentes rubros que componen HCF, USPYSF y HC.

Tabla 3. Constructo de variables para la conformación del modelo.

\begin{tabular}{|l|l|l|}
\hline \multicolumn{1}{|c|}{ HCF } & \multicolumn{1}{|c|}{ USPYSF } & \multicolumn{1}{c|}{ HC } \\
\hline $\begin{array}{l}\text { Principales acciones que realiza } \\
\text { al recibir su ingreso }\end{array}$ & $\begin{array}{l}\text { Número de cuentas bancarias } \\
\text { de ahorro }\end{array}$ & $\begin{array}{l}\text { Conocimientos de los términos } \\
\text { y condiciones de las tarjetas de } \\
\text { crédito }\end{array}$ \\
\hline $\begin{array}{l}\text { Gastos que genera } \\
\text { frecuentemente }\end{array}$ & Ahorros & Propósitos del seguro de vida \\
\hline
\end{tabular}




\begin{tabular}{|c|c|c|}
\hline Presupuestación de gastos & Número de tarjetas de crédito & $\begin{array}{l}\text { Conocimientos del pago de } \\
\text { impuestos en México }\end{array}$ \\
\hline $\begin{array}{l}\text { Frecuencia de problemas para } \\
\text { pagar los servicios básicos }\end{array}$ & $\begin{array}{l}\text { Número de veces que usa la } \\
\text { tarjeta de crédito al mes }\end{array}$ & Pago de impuestos \\
\hline $\begin{array}{l}\text { Porcentaje que dirige de su } \\
\text { ingreso al ahorro }\end{array}$ & $\begin{array}{l}\text { Cantidad que paga en su tarjeta } \\
\text { de crédito al mes }\end{array}$ & $\begin{array}{lll}\begin{array}{l}\text { Conceptos } \\
\text { conocidos }\end{array} & \text { que resultan } \\
\end{array}$ \\
\hline $\begin{array}{l}\text { Tiempo que puede solventarse } \\
\text { económicamente ante la falta } \\
\text { del ingreso principal }\end{array}$ & $\begin{array}{l}\text { Formas en las que paga sus } \\
\text { vacaciones }\end{array}$ & $\begin{array}{l}\text { Conocimientos que tiene sobre } \\
\text { las instituciones financieras }\end{array}$ \\
\hline $\begin{array}{l}\text { Porcentaje que destina de su } \\
\text { ingreso a necesidades básicas }\end{array}$ & $\begin{array}{l}\text { Productos de ahorro o inversión } \\
\text { para el retiro }\end{array}$ & Nivel de habilidad en idiomas \\
\hline Pasatiempos & $\begin{array}{l}\text { Servicios de protección con los } \\
\text { que cuenta }\end{array}$ & $\begin{array}{l}\text { Nivel de conocimientos en } \\
\text { herramientas computacionales }\end{array}$ \\
\hline $\begin{array}{l}\text { Porcentaje que destina de su } \\
\text { ingreso al entretenimiento }\end{array}$ & Fondo de emergencia & Problema de interés simple \\
\hline $\begin{array}{l}\text { Porcentaje que destina al pago } \\
\text { de deudas }\end{array}$ & $\begin{array}{l}\text { Uso de aplicaciones } \\
\text { tecnológicas para gastar, } \\
\text { invertir o ahorrar }\end{array}$ & Problema de Interés compuesto \\
\hline $\begin{array}{l}\text { Motivos por el cual adquiere } \\
\text { deudas }\end{array}$ & & Problemas de Inflación \\
\hline $\begin{array}{l}\text { Emoción que experimenta al } \\
\text { momento de realizar sus } \\
\text { compras }\end{array}$ & & Problema de Riesgo \\
\hline $\begin{array}{l}\text { Momentos en los que compra } \\
\text { regalos }\end{array}$ & & Inversión \\
\hline
\end{tabular}

Fuente: Elaboración propia con base en la EFIES (Hernández-Rivera, 2020).

El modelo propuesto tuvo un Alfa de CronBach de 0.761 (ver tabla 4), que de acuerdo con la literatura el modelo tiene validación, resulta adecuado, ya que estos valores aceptables (Hernández y Barrera, 2017; Bojórquez, López, Hernández y Jiménez, 2013). Después de revisar la estadística de fiabilidad, se realizó un análisis de regresión múltiple para observar la relación e incidencia entre las variables.

Tabla 4. Estadística de fiabilidad.

\begin{tabular}{|c|c|}
\hline $\begin{array}{c}\text { Alfa de } \\
\text { CronBach }\end{array}$ & $\begin{array}{c}\text { N. de } \\
\text { elementos }\end{array}$ \\
\hline .761 & 4 \\
\hline
\end{tabular}

Fuente: Elaboración propia con ayuda de software estadístico SPSS. 


\subsection{Análisis de Resultados}

Tabla 5. Prueba ANOVA a.

\begin{tabular}{|c|l|r|r|r|r|}
\hline \multicolumn{2}{|c|}{ Modelo } & $\begin{array}{c}\text { Suma de } \\
\text { cuadrados }\end{array}$ & \multicolumn{1}{c|}{ gl } & \multicolumn{1}{c|}{$\begin{array}{c}\text { Media } \\
\text { cuadrática }\end{array}$} & \multicolumn{1}{c|}{ Sig. } \\
\hline \multirow{2}{*}{1} & Regresión & 339617.495 & 3 & 113205.837 & $.000^{\mathrm{b}}$ \\
\cline { 2 - 6 } & Residuo & .000 & 3597 & .000 & \\
\cline { 2 - 7 } & Total & 339617.495 & 3600 & & \\
\hline
\end{tabular}

Fuente: Elaboración propia con ayuda de software estadístico SPSS.

a. Variable dependiente: Scoring IF

b. Predictores: (Constante), HCF, HC, USPYSF

\section{Si el Valor $p \leq \alpha$ : El modelo econométrico es estadísticamente significativo.}

Analizando el valor de F se puede concluir que el modelo es estadísticamente significativo, ya que la significación asintótica (Pi-Value) es menor que .05

Tabla 6. Coeficientes a.

\begin{tabular}{|c|c|c|c|c|c|}
\hline \multirow{2}{*}{\multicolumn{2}{|c|}{ Modelo }} & \multicolumn{2}{|c|}{$\begin{array}{l}\text { Coeficientes no } \\
\text { estandarizados }\end{array}$} & \multirow{2}{*}{$\begin{array}{c}\text { Coeficientes } \\
\text { estandarizados } \\
\text { Beta }\end{array}$} & \multirow{3}{*}{$\begin{array}{l}\text { Sig. } \\
\\
.000\end{array}$} \\
\hline & & B & Error & & \\
\hline \multirow[t]{4}{*}{1} & (Constante) & $-8.882 \mathrm{E}-14$ & .336 & & \\
\hline & HCF & 1.000 & .045 & .580 & .000 \\
\hline & USPYSF & 1.000 & .069 & .434 & .000 \\
\hline & $\mathrm{HC}$ & 1.000 & .014 & .447 & .000 \\
\hline
\end{tabular}

Fuente: Elaboración propia con ayuda de software estadístico SPSS.

a. Variable dependiente: Scoring IF.

Se puede notar que el modelo econométrico es de la forma:

\section{Scoring inclusión financiera = -.8,8E-14+.580HCF+ .434USPYSF+.447HC}

El coeficiente HCF es mayor a HC (.447), seguido de USPYSF (.434). Se puede señalar que, ante un aumento porcentual en los hábitos y comportamientos financieros conlleva a un mejor puntaje en inclusión financiera.

Tabla 7. Prueba de KMO y Bartlett.

\begin{tabular}{|l|r|c|}
\hline \multicolumn{2}{|l|}{ Media Kaiser-Meyer-Olkin de adecuación de muestreo } & .501 \\
\hline $\begin{array}{l}\text { Prueba de esfericidad de } \\
\text { Bartlett }\end{array}$ & Aprox. Chi-cuadrado & 2923.969 \\
\cline { 2 - 3 } & gl & 3 \\
\cline { 2 - 3 } & Sig. & .000 \\
\hline
\end{tabular}

Fuente: Elaboración propia con ayuda de software estadístico SPSS. 
Aplicando la prueba de Bartlett se observa que existe significancia bilateral por lo que se concluye que hay homogeneidad de las varianzas.

El siguiente cuadro categoriza el puntaje de inclusión financiera por estado, de las localidades analizadas en las 5 principales zonas metropolitanas de México. A mayor Inclusión Financiera menor Scoring (donde 1 es lo mejor y 5 es lo peor). Es decir, entre menor puntuación o Scoring mayor es el grado de inclusión financiera que tienen los Jóvenes Universitarios de las Zonas Metropolitanas consideradas en el estudio.

Tabla 8. Inclusión Financiera por Estado.

\begin{tabular}{|c|c|c|c|c|c|}
\hline $\begin{array}{c}\text { Zona } \\
\text { Metropolitana } \\
\text { Monterrey } \\
\text { (Nuevo León) }\end{array}$ & $\begin{array}{c}\text { Metropolitana } \\
\text { Guadalajara } \\
\text { (Jalisco) }\end{array}$ & $\begin{array}{c}\text { Metropolitana } \\
\text { Valle de México }\end{array}$ & $\begin{array}{c}\text { Metropolitana } \\
\text { (Ciudad de } \\
\text { Toluca } \\
\text { (Estado de } \\
\text { México) }\end{array}$ & \multicolumn{2}{|c|}{$\begin{array}{c}\text { Zona Metropolitana } \\
\text { Puebla - Tlaxcala } \\
\text { (Puebla) }\end{array}$} \\
\hline $\mathbf{2 6 . 1 7 8 5 6 3}$ & $\mathbf{2 9 . 7 6 5 9 8 7}$ & $\mathbf{3 2 . 7 8 9 8 6 7}$ & $\mathbf{3 5 . 3 4 6 7 8 8}$ & $\mathbf{3 9 . 8 9 6 7 4 3}$ & $\mathbf{4 1 . 4 5 6 7 8 2}$ \\
\hline
\end{tabular}

Fuente: Elaboración propia con ayuda de software estadístico SPSS.

La escala likert está conformada por categorías de forma descendente, en el modelo se consideraron todas las categorías que contemplan la inclusión financiera. Los resultados del Scoring se muestran en la tabla 8, concluyendo que la Zona Metropolitana con mayor Inclusión Financiera es Monterrey en el Estado de Nuevo León, dado que presenta la menor puntuación en el Scoring. El caso opuesto es la Zona Metropolitana Puebla-Tlaxcala que presenta la mayor puntuación, es decir la menor inclusión financiera.

Esta investigación junto a otras similares (Osorno y Hernández-Rivera, 2021), coinciden que los jóvenes universitarios aún no logran incluirse de manera formal al sistema financiero, incluso en aquellas zonas donde el desarrollo bancario y los puntos de acceso están disponibles para la mayoría de la población. A través de un modelo scoring se estimó la categorización de la inclusión financiera en los diferentes estados y zonas metropolitanas de México a través de un análisis estadístico y econométrico. Basándose en diversos estudios (Ochoa, Galeano, Agudelo, 2010; Rayo, Lara y Camino 2010), se muestra al scoring como un puntaje de una expresión numérica sustentada en un análisis del nivel de datos de una muestra, que indica directamente la probabilidad de que el resultado sea favorable o no.

\section{Conclusiones.}

La investigación presentó un modelo econométrico scoring para categorizar el puntaje de inclusión financiera y sus determinantes, considerando lo siguiente: a) hábitos y comportamientos financieros, b) uso de servicios y productos financieros, así como c) habilidades y conocimientos que tienen los jóvenes universitarios. Se encontraron algunas diferencias significativas para cada caso. 
Los estudiantes pertenecientes a las zonas metropolitanas con un mayor desarrollo económico como Monterrey, Guadalajara y Valle de México, en su mayoría obtuvieron las mejores categorías de inclusión, lo que se demuestra que esta población estudiantil cuenta con un mejor nivel de educación; así también mayores puntos de acceso al mercado financiero. Caso contrario a las zonas metropolitanas de Toluca y Puebla -Tlaxcala donde los niveles de inclusión no son los óptimos, así como la falta de educación financiera y puntos de acceso.

El presente estudio abarcó aspectos económicos, financieros, educativos, geográficos y sociales; ya que se analizó la inclusión en los jóvenes desde la perspectiva de estas diciplinas. Por otra parte, se encontró que los jóvenes universitarios aún no están siendo incluidos, en aquellas zonas con mayores oportunidades, por lo que existe una posible "desigualdad financiera".

Las medidas adoptadas por el Gobierno Federal y la iniciativa privada para incluir a la población joven al mercado financiero en México dan resultado, pero no son suficientes. Esto se debe a varios factores como la ubicación geográfica, el nivel económico, desarrollo, educación, oportunidades, empleo, entre otros. Así como los rezagos y deficiencias con los que enfrenta el Sistema Financiero Mexicano.

La información y categorización de inclusión financiera, permite conocer el comportamiento económico de los universitarios en México, analizando su comprensión y participación en el mercado. En el estudio se expusieron diversas variables para observar la toma de decisiones de los jóvenes universitarios con la finalidad de que este material sirva de precedente para la propuesta de programas, políticas, material educativo, que permita que este grupo de la población cada vez esté menos excluido.

Adicionalmente este trabajo, puede propiciar que existan nuevas investigaciones enfocadas a este sector de la población en particular, ya que, si los universitarios que estudian temas económicosfinancieros no son capaces de incluirse financieramente, probablemente será más complicado para otras áreas del conocimiento. Sin embargo, sería benéfico que las Universidades fomentaran la inclusión financiera en toda la población estudiantil sin importar la carrera que cursan, considerando que cuando están participando en el mercado se genera dinamismo económico para el país. Por ello, es necesario brindarles a los jóvenes, las herramientas necesarias para ser parte del sistema financiero de México, así como la educación suficiente para que realicen adecuado uso de los servicios y productos ofertados por las entidades financieras.

\section{Referencias}

[1] América Economía Inteligente, (2018), Mejores Universidades de México Rankings 2018, MBA América Economía, Recuperado en: https://mba.americaeconomia.com/articulos/notas/conozca-losresultados-del-ranking-de-universidades-de-mexico-2019

[2] Arango E. L. y Cardona Sosa L., (2019), Tarjetas de crédito en personas de ingresos medios y bajos en Colombia ¿qué determina su uso?, Borradores de Economía, (1089), 3-6.

[3] Argüello Segovia A. C., (2018), Análisis del Impacto Financiero en los seguros de asistencia médica por la implementación de la ley orgánica de medicina prepagada en Ecuador (tesis de carrera), Pontifica Universidad Católica del Ecuador-Matriz, Quito, Ecuador. 
[4] Asociación Nacional de Universidades e Instituciones de Educación Superior, ANUIES, (2020). Anuarios Estadísticos de Educación Superior. Recuperado de http://www.anuies.mx/informacion-yservicios/informacion-estadistica-de-educacion-superior/anuario-estadistico-de-educacion-superior

[5] Banco de México, BANXICO (2019), CoDI: la nueva forma de pagar en México, Recuperado en: https://www.banxico.org.mx/publicaciones-y-prensa/presentaciones/\%7B1CA33D18-A38C-EE2941BF-6302A641D617\%7D.pdf

[6] Banco de México, Banxico (2020), Plan De Consulta Para El Proyecto De Disposiciones En Materia De Cuentas De Depósitos Para Menores De Edad Y Montos Máximos Provisionales Para Cuentas Nivel 2. Recuperado de file:///C:/Users/skind/AppData/Local/Temp/MicrosoftEdgeDownloads/09a6152e2f13-4d25-a597-3fda5e5e6f80/\%7BEED65C5C-EA44-ACB2-5898-6407851153EB\%7D\%20(3).pdf

[7] Banco Interamericano de Desarrollo, BID (2015), Integración e inclusión financiera; movilizando recursos para el desarrollo social y económico, II Cumbre Empresarial de la Américas, 3-4. Recuperado de: $\quad$ https://publications.iadb.org/es/publicacion/15450/integracion-en-inclusion-financieramovilizando-recursos-para-el-desarrollo

[8] Banco Mundial, BM (2018). Inclusión Financiera. Banco Mundial BIRF AIF. Recuperado de: https://www.bancomundial.org/es/topic/financialinclusion/overview

[9] Bermeo Giraldo M. C., Álvarez Agudelo L., Ospina Rúa M. I., Acevedo Correa Y. y Montoya Restrepo I. A., (2019), Factores que influyen en la intención de uso de las tarjetas de crédito por parte de los jóvenes universitarios. Revista CEA, 5(9), 77-96. doi:| org/10.22430/24223182.1257

[10] Bojórquez Molina J., López Aranda L., Hernández Flores M., Jiménez López E., (2013), Utilización del alfa de Cronbach para validar la confiabilidad de un instrumento de medición de satisfacción del estudiante en el uso del software Minitab, (LACCEI'2013).

[11] Ceballos Mina O. E. y Santiago Ayala L. E., (2019), Efectos de las tarjetas de crédito en la estructura de consumo y la desigualdad de los hogares mexicanos, Revista Finanzas y Política Económica, 11 (2), 301305.

[12] Centro de Estudios de las Finanzas Públicas, 2018. Boletín Financiero Mexicano. Recuperado de https://www.cefp.gob.mx/publicaciones/boleco/2018/becefp0212018.pdf

[13] Citibanamex, Índice de Inclusión Financiera, 2019. Recuperado de https://www.banamex.com/sitios/analisis-

financiero/pdf/Economia/20191023INFORMECITIBANAMEX.pdf

[14] Comisión Nacional Bancaria y de Valores, 2019, Boletín de Inclusión Financiera, Recuperado de: https://www.gob.mx/cms/uploads/attachment/file/448668/Bolet_n_IF.pdf

[15] Comisión Nacional Bancaria y de Valores, 2021, El ahorro en México: productos, instrumentos y evolución (con datos de la Encuesta Nacional de Inclusión Financiera), Recuperado de: https://www.gob.mx/cms/uploads/attachment/file/616136/Estudio_Ahorro.pdf

[16] Comisión Nacional Bancaria y de Valores, CNBV (2016), Inclusión Financiera, Recuperado de: https://www.gob.mx/cnbv/acciones-y-programas/inclusion-financiera-25319

[17] Comisión Nacional Bancaria y de Valores, CNBV (2018), Resultados de la Encuesta Nacional de Inclusión Financiera \#ENIF 2018, 3-4, Recuperado de: https://www.gob.mx/cms/uploads/attachment/file/414831/Cuadr_ptico_2018_verimpresa.pdf

[18] Comisión Nacional del Mercado de Valores y Banco de España, (2010), Plan de Educación Financiera, Finanzas para Todos, Recuperado de: https://www.finanzasparatodos.es/es/comollegarfindemes/fondoemergencia/cuantohayquetener.h tml

[19] Comisión Nacional para la Protección y Defensa de los Usuarios de Servicios Financieros, CONDUSEF (2018), Educación e Inclusión Financiera en México, 18-21, Recuperado de: https://www.condusef.gob.mx/Revista/PDF-s/2019/227/educacion.pdf

[20] Comisión Nacional para la Protección y Defensa de los Usuarios de Servicios Financieros, CONDUSEF (2019a), Conoce la nueva plataforma de cobro digital, Recuperado de: https://www.condusef.gob.mx/Revista/PDF-s/2019/228/codi.pdf 
[21] Comisión Nacional para la Protección y Defensa de los Usuarios de Servicios Financieros, CONDUSEF (2019b), Asegura el futuro de tus hijos, Gobierno de México, Recuperado de: https://www.google.com/url?sa=t\&source=web\&rct=j\&url=https://www.condusef.gob.mx/docume ntos/464006_20_Consejos_para_tu_bolsillo..._Seguro_educativo.pdf\&ved=2ahUKEwjDtNzZlLTpAhWD Hc0KHQNFBp0QFnoECAgQAA\&usg=AOvVaw1yGlnA5V9YeO0XzY_pQqut

[22] Consejo Nacional de Inclusión Financiera, CNIF (2016), Política Nacional de Inclusión Financiera 4, Recuperado de: file:///C:/Users/lecg9/Downloads/PNIF_ver_1jul2016CONAIF_vfinal.pdf

[23] Consejo Nacional de Inclusión Financiera, CNIF (2017), Reporte Nacional de Inclusión Financiera 8, 14-15, Recuperado de: https://www.cnbv.gob.mx/Inclusi\%C3\%B3n/Documents/Reportes\%20de\%20IF/Reporte\%20de\% 20Inclusion\%20Financiera\%208.pdf

[24] Consejo Nacional de Inclusión Financiera, CNIF (2018), Reporte Nacional de Inclusión Financiera 9, 66-70, Recuperado de: https://www.cnbv.gob.mx/Inclusi\%C3\%B3n/Documents/Reportes\%20de\%20IF/Reporte\%20de\% 20Inclusion\%20Financiera\%209.pdf

[25] Consejo Nacional de Población, 2018, Proyecciones de la Población de México y de las Entidades Federativas, 2016-2050 y Conciliación Demográfica de México, 1950 -2015, Recuperado de: https://www.gob.mx/cms/uploads/attachment/file/503374/Diccionario_Proyecciones_de_la_Pobla cion_2016_2050.pdf

[26] Díaz Caicedo 0. A., (2020), Análisis de los factores que influyen en el uso de las plataformas digitales en la banca ecuatoriana por los millennials (tesis de carrera), Universidad Católica de Santiago de Guayaquil, Guayaquil, Ecuador.

[27] Escalera Chávez M. E., Tejada Peña E., García Santillán A., (2017), Uso de los servicios financieros; Estudio Empírico en los Estudiantes Universitarios, 2 (1), 581-590.

[28] Fajardo C., Camilla Castillo M., Uribe J. y Paipa L. C., (2018), Cubrir riesgos, decisión de compra de seguro de hogar (trabajo de grado), Universidad EAN, Bogotá, Colombia.

[29] Guimaraes Vélez V. S., (2020), Factores que impulsan la desdolarización en el Perú (tesis de licenciatura), Universidad del Pacifico, Lima, Perú.

[30] Guízar I., González-Vega C. y Miranda M. J., (2015), Un análisis numérico de inclusión financiera y pobreza, EconoQuantum, 12 (2), 7-21.

[31] Hernández Andrés H., Barrera Pascual A., (2017), Validación de un instrumento de investigación para el diseño de una metodología de autoevaluación del sistema de gestión ambiental, 9 (1), 157-163, doi: https://doi.org/10.22490/21456453.2186

[32] Hernández Rivera A., Rendón Rojas L., (2020), Brecha de género tecnológica en la educación financiera universitaria en México, 26 (93), 48-64.

[33] Hernández-Rivera, Ariadna, (2019), Educación financiera en la educación superior: estudio de 19 universidades en México, 218, 39-49.

[34] Hernández Rivera Ariadna, (2020), Educación Financiera en los Jóvenes Universitarios de México, Ciudad de México, Editorial Educación y Cultura.

[35] Hernández Rivera, Ariadna. (2019). Educación financiera en la educación superior: estudio de 19 universidades en México. Universidad Autónoma Metropolitana. El Cotidiano Revista de la realidad mexicana actual, Año. 35, No. 218, México, Universidad Autónoma Metropolitana, pp.39-48.

[36] Home Salazar E., (2016), El microcrédito como herramienta de emprendimiento: un enfoque desde la educación financiera en Colombia (tesis de maestría), Universidad Militar Nueva Granada, Bogotá, Colombia.

[37] Hoyo, M. C., Pena, H. X., y Tuesta, D. (2013), Factores de demanda que influyen en la Inclusión Financiera en México: Análisis de las barreras a partir de la ENIF. BBVA, 2-3.

[38] Instituto Mexicano de la Juventud, (2020). Las personas jóvenes en México representan casi un tercio de la población total: CONAPO e IMJUVE, Recuperado de: https://www.gob.mx/imjuve/articulos/las- 
personas-jovenes-en-mexico-representan-casi-un-tercio-de-la-poblacion-total-conapo-eimjuve?idiom=es

[39] Instituto Nacional de Estadística y Geografía, 2018, Encuesta Nacional de la Dinámica Demográfica ENADID 2018, Recuperado de: https://www.inegi.org.mx/contenidos/programas/enadid/2018/doc/resultados_enadid18.pdf

[40] Instituto Nacional de Estadística y Geografía, 2018, Estadísticas a propósito del día mundial de la población (11 de julio) datos nacionales, Recuperado de: https://www.inegi.org.mx/contenidos/saladeprensa/aproposito/2020/Poblacion2020_Nal.pdf

[41] Instituto Nacional de Estadística y Geografía, 2020, Información de México para niños, Recuperado de: http://cuentame.inegi.org.mx/poblacion/habitantes.aspx?tema=P\%20]

[42] Klapper, L. \& Hess, J. (2016). Financial Inclusion and Women's Economic Empowerment. A Briefing for the un-Secretary-General's High-Level Panel on Women's Economic Empowerment. The World Bank, Development Research Group. Finance \& Private Sector Development Team.

[43] Lecuona Valenzuela R., (2017), Instrumentos para la inclusión financiera: el caso de México. Casos exitosos de instrumentos de financiamiento para las pymes de la banca de desarrollo en México, Naciones Unidas, 15, Recuperado de: https://repositorio.cepal.org/bitstream/handle/11362/42504/1/S1700631_es.pdf

[44] Ley de Ahorro y Crédito Popular, Diario Oficial de la Federación de los Estados Unidos Mexicanos, 04 de junio de 2001.

[45] Ley para el Programa Nacional de Juventud 2014-2018, Diario Oficial de la Federación de los Estado Unidos Mexicanos, 30 de abril 2014.

[46] Li. N., Espinosa. A., Cárdenas. G. y Serrano C. (2018), ENIF 2018: 63.2\% de los mexicanos usan ahorro informal, 70.2\% usan créditos informales. BBVA Research. 1-3.

[47] Lusardi A. y Mitchell S. O., (2011), Financial Literacy around the world an overview, NBER Working Paper Series, (17107), 7-8.

[48] Morales Ramírez M. A., (2019), La reforma al sistema de ahorro para el retiro en México: ¿Es progresista?, Cielo Laboral, 1-2, Recuperado de: https://www.google.com/url?sa=t\&source=web\&rct=j\&url=http://www.cielolaboral.com/wpcontent/uploads/2019/03/morales_ramirez_noticias_cielo_2019_03.pdf\&ved=2ahUKEwjZgLzVjLTp AhUHCc0KHVMODeMQFjAAegQIAxAB\&usg=AOvVaw3K6Q0ASGEQKqsvXARCtx16

[49] OCDE/CAF/CEPAL (2018), Perspectivas económicas de América Latina 2018: Repensando las instituciones para el desarrollo, Editions OCDE, Paris, 76-80.

[50] Ochoa P., Galeano M., Agudelo V., (2010), Construcción de un modelo de Scoring para el otorgamiento de crédito en una entidad financiera, (16), 191-222.

[51] Oficina de Información Científica y Tocológica para el Congreso de la Unión, INCyTU (2018), Educación financiera en México, (2018), 1-4, Recuperado de: https://www.google.com/url?sa=t\&source=web\&rct=j\&url=https://foroconsultivo.org.mx/INCyTU/ documentos/Completa/INCYTU_18-018.pdf\&ved=2ahUKEwi4jZhx7HpAhWOZM0KHU0oBcQQFnoECAcQAA\&usg=AOvVaw3kiMXjw7HiNj0gxpm7s_EN

[52] Ordaz Hernández M. B., Pacheco B. A., Guillén A. A. y Gutiérrez Rodríguez J. A., (2019), Percepción de la importancia del ahorro para el retiro en los estudiantes de la Universidad Veracruzana. Revista Internacional La Nueva Gestión Organizacional, (11), 129-154.

[53] Osorno Morales L., Hernández Rivera A., (2021), Las desigualdades de género en La educación financiera universitaria en México 2017-2018, 8, 18-40.

[54] Peterson K., O., (2018), Impact of digital finance on financial inclusion and stability, Borsa Istanbul Review, 18 (4), 329-340.

[55] Procuraduría Federal del Consumidor, (2017), Los Jóvenes y las Finanzas, Recuperado de: https://www.gob.mx/profeco/documentos/los-jovenes-y-las-finanzas?state=published

[56] Raccanello K., y Herrera Guzmán E., (2014), Educación e Inclusión Financiera, Revista Latinoamericana de Estudios Educativos (México), 44 (2), 122-124. 
[57] Rayo Cantón S., Lara Rubio J., Camino Blasco D., (2010), Un Modelo de Credit Scoring para instituciones de microfinanzas en el marco de Basilea II, 15 (28), 89-124.

[58] Rincón Higuera A. L., (2019), Límites a la práctica de compensación bancaria en los contratos de depósito de cuenta de ahorros en modalidad de nómina como garantía de derechos mínimos de los consumidores financieros categorizados como 'trabajadores'. Un análisis de derecho colombiano, $\begin{array}{lllll}\text { Revista Estudios } & \text { Socio-Jurídicos, } & 22 & \text { (1), 295-297. }\end{array}$ doi:|org/10.12804/revistas.urosario.edu.co/sociojuridicos/a.7630

[59] Rivera Ochoa B., Bernal Domínguez D., 2018, La importancia de la educación financiera en la toma de decisiones de endeudamiento. Estudio de una sucursal de "Mi Banco" en México, Departamento de Administración, Economía y Finanzas, 21 (41), 117-144.

[60] Roa M. J. (2013). Inclusión financiera en América Latina y el Caribe: acceso, uso y calidad. Boletín del Centro de Estudios Monetarios Latinoamericanos CEMLA, 121-122.

[61] Rochman Ratner R., (2012), Análisis del Credit Scoring, 53 (3), 303-315.

[62] Salazar Cantú J. J., Rodríguez Guajardo R. C. y Jaramillo Garza J., (2017), Inclusión financiera y cohesión social en los municipios de México, Revista Mexicana de Economía y Finanzas Nueva Época, 12 (3), 4566. Doi: https://doi.org/10.21919/remef.v12i3.96

[63] Sánchez González M., (2013). La tarea compartida de la inclusión financiera, Educa Banxico, Recuperado de: http://educa.banxico.org.mx/pdfs/discursos/\%7B18F50800-C79D-81F7-F912198A7E3D7B7C\%7D.pdf

[64] Sarma M. y Pais J., (2010), Financial Inclusion and Development, Journal of International Development, 23, 613-628. doi:| 10.1002/jid.1698

[65] Schröeder C., (2016), Educación financiera: ¿y por qué?, Revista eXtoikos, (18), 41-42.

[66] Secretaría de Desarrollo Agrario Territorial y Urbano (SEDATU), Consejo Nacional de Población (CONAPO) e Instituto Nacional de Estadística y Geografía (INEGI), (2018), Delimitación de las zonas Metropolitanas 2015, 3-20, Recuperado de: https://www.inegi.org.mx/app/biblioteca/ficha.html?upc=702825006792

[67] Secretaría de Gobernación / Secretaría General del Consejo Nacional de Población; Secretaría de Desarrollo Agrario, Territorial y Urbano, 2018, Sistema Urbano Nacional 2018, Recuperado de: file://C:/Users/lecg9/Downloads/SUN_2018_vgM3fWA.pdf

[68] Secretaría del Trabajo y Previsión Social, STPS (2019), Preguntas Frecuentes / Jóvenes Construyendo el Futuro, Recuperado de: https://www.gob.mx/stps/articulos/preguntas-frecuentes-jovenesconstruyendo-el-futuro

[69] Vargas Hernández L. A. y Reyes Hernández M. R., (2019). Visiones de la Educación Financiera: análisis y perspectivas. Hernández-Rivera A. y Pérez Munguía S. Coordinadoras. Ed. BUAP. P.33.

[70] Vera Lezama Y. A., (2019), Desarrollo e Implementación de una aplicación móvil para la gestión de seguros de autos en Pacífico Seguros utilizando Tensorflow (tesis de carrera), Universidad Tecnológica del Perú. Lima, Perú.

[71] Villacorta O. y Reyes J. D., (2012), Servicios financieros para las mayorías, La inclusión financiera en México. Revista MBS. (2). 10-11.

[72] Zapata Aguilar A., Cabrera Ignacio E., Hernández Arce J. y Martínez Morales J., (2016), Educación financiera entre jóvenes universitarios: Una visión general, Revista Administración y Finanzas, 3 (9), 27. 